\title{
Thin and thick cloud top height retrieval algorithm with the Infrared Camera and LIDAR of the JEM-EUSO Space Mission
}

\author{
G. Sáez-Cano ${ }^{1, a}$, J.A. Morales de los Ríos ${ }^{1}$, L. del Peral ${ }^{1,2}$, A. Neronov ${ }^{2}$, S. Wada $^{3}$, \\ and M.D. Rodríguez Frías ${ }^{1,2}$ for the JEM-EUSO Collaboration \\ ${ }^{1}$ Space \& Astroparticle Group, UAH, Madrid, Spain \\ 2 ISDC, Astronomy Dept. University of Geneva, Switzerland \\ ${ }^{3}$ RIKEN Advanced Science Institute, Japan
}

\begin{abstract}
The origin of cosmic rays have remained a mistery for more than a century. JEM-EUSO is a pioneer space-based telescope that will be located at the International Space Station (ISS) and its aim is to detect Ultra High Energy Cosmic Rays (UHECR) and Extremely High Energy Cosmic Rays (EHECR) by observing the atmosphere. Unlike ground-based telescopes, JEM-EUSO will observe from upwards, and therefore, for a properly UHECR reconstruction under cloudy conditions, a key element of JEM-EUSO is an Atmospheric Monitoring System (AMS). This AMS consists of a space qualified bi-spectral Infrared Camera, that will provide the cloud coverage and cloud top height in the JEM-EUSO Field of View (FoV) and a LIDAR, that will measure the atmospheric optical depth in the direction it has been shot. In this paper we will explain the effects of clouds for the determination of the UHECR arrival direction. Moreover, since the cloud top height retrieval is crucial to analyze the UHECR and EHECR events under cloudy conditions, the retrieval algorithm that fulfills the technical requierements of the Infrared Camera of JEM-EUSO to reconstruct the cloud top height is presently reported.
\end{abstract}

\section{Introduction}

Although cosmic rays have been discovered over one century ago, still their origin remains unknown. JEMEUSO (Extreme Universe Space Observatory on-board the Japanese Experiment Module) is a pioneer space-based experiment meant to detect Ultra High Energy Cosmic Rays (UHECR) and Extremely High Energy Cosmic Rays (EHECR). It will observe the Extensive Air Showers (EAS) produced in the atmosphere when the UHECR and EHECR interact with the atmospheric nuclei.

To estimate the physical properties of the UHECR such as energy, arrival direction and its composition, the EAS fluorescence light profile and the EAS Cherenkov light profile has to be measured. Since JEM-EUSO will cover a very large observation area in the atmosphere $\left(\sim 10^{5} \mathrm{~km}^{2}\right)$, a properly monitoring of the atmospheric conditions is mandatory. The Atmospheric Monitoring System (AMS) $[1,2]$ of JEM-EUSO will consist of a bispectral and space qualified Infrared Camera and a LIDAR (LIght Detection And Ranging). The bi-spectral IR camera gives the cloud coverage in the FoV of the main telescope. With the difference in the brightness temperature in the IR bands and a radiative retrieval model, the clouds altitude is provided. Thanks to the laser back-scatter signal, the optical depth $(\tau)$ profiles of the atmosphere in selected directions are measured (detecting aerosol and cloud layers).

To increase the statistics of the detected EAS, JEMEUSO may use certain triggered events observed in cloudy scenarios [3]. The main criteria to classify cloudy events as "reconstructible events" is the detection of the depth of maximum development. The shower maximum will be

\footnotetext{
a e-mail: lupe.saez@uah.es
}

observable in cloudy conditions if $\tau<1$ (the cloud is optically thin) or if $H_{\max }>H_{c}$ (i.e., the altitude of the shower maximum is higher than the cloud top height) $[4,5]$.

In this work we focus into two main features of the EAS reconstruction in cloudy conditions: the EAS geometry analysis and the reconstruction of the cloud top height.

\section{Shower geometry in cloudy conditions}

EAS signal is detected as a spot moving on the focal surface of the JEM-EUSO main telescope. To analyse the direction of the incoming shower, the direction can be understood as the composition of two projections: the azimutal angle, contained in the focal plane, and the zenith angle, contained in the track detector plane (the plane which contains the shower track and the detector). The former is calculated with the projection of the image of the shower in the focal surface. The latter, from the timing information and arrival angle of the EAS photons to the detector [6].

The timing fit method is based on the viewing angle $(\alpha)$, which is the angle between the direction given by two pixels, and the arrival time $\left(\zeta_{i}\right)$. The zenith angle $(\theta)$ can be calculated thanks to this timing fit (Fig. 1):

where $D_{J E}$ is the distance from JEM-EUSO to the ground, $D_{1}$ is the distance between the beginning of the shower to the telescope, $D_{2}$ is the distance from the telescope to the EAS core, $\mathrm{L}$ is the shower length, $\gamma$ is the angular distance between the center of the FoV and the EAS core, and $t_{i}$ is the time at which the photons have

This is an Open Access article distributed under the terms of the Creative Commons Attribution License 4.0, which permits unrestricted use, distribution, and reproduction in any medium, provided the original work is properly cited. 


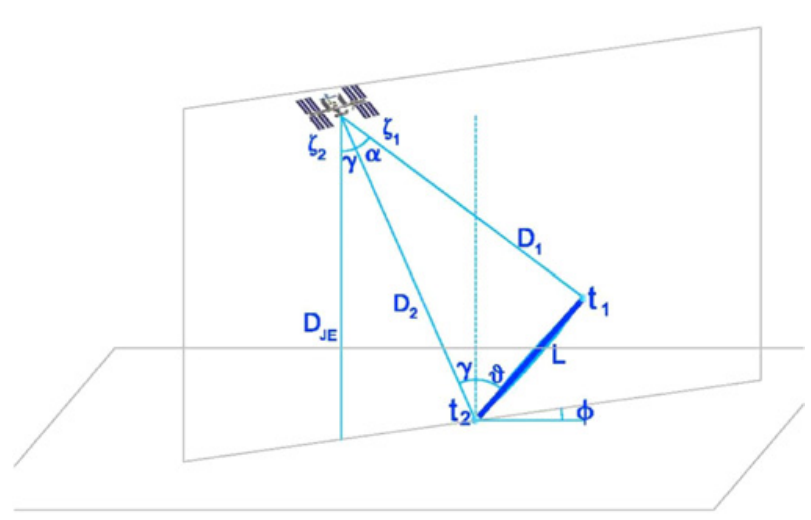

Figure 1. Scheme of the geometrical relations used for the shower geometry.

been created. From the scheme 1 it is clear that:

$$
D_{2}=\frac{D_{J E}}{\cos (\gamma)} \text {. }
$$

For simplicity we assume that the first EAS photon is emitted at $t_{1}=0$ seconds. Then, the relation between the time and the zenith angle will be given by:

$$
\left(\zeta_{1}-\zeta_{2}\right)=\frac{D_{2}+l-D_{1}}{c}
$$

Which can be expressed as a function of $\gamma, \alpha$ and $\theta$ :

$$
\left(\zeta_{1}-\zeta_{2}\right)=\frac{1}{c} \cdot \frac{D_{J E}}{\cos (\gamma)} \cdot\left(1+\frac{\sin (\alpha)-\sin (\gamma+\theta)}{\sin (\alpha+\gamma+\theta)}\right) \text {. }
$$

For showers landing on the center of the $\operatorname{FoV}(\gamma=0)$, this equation is simplified to Eq. (4).

$$
\left(\zeta_{1}-\zeta_{2}\right)=\frac{D_{J E}}{c} \cdot\left(1+\frac{\sin (\alpha)-\sin (\theta)}{\sin (\alpha+\theta)}\right) .
$$

In Fig. 2 it has been represented the viewing angle as a function of the arrival time for a proton induced shower with a zenith angle of $60^{\circ}$ and an azimutal angle of $45^{\circ}$ landing on the center of the FoV in three different cases: in clear atmosphere (upper panel), in presence of an optically thin cloud (intermediate panel), and in presence of an optically thick cloud whose cloud top height is slightly below the shower maximum (lower panel). The signal has been fitted with the model 4 . For the optically thin cloud case, even if some few photons have been scattered due to the cloud and their trajectory has changed, the track of the shower is still well defined. On the other hand, in the case of the optically thick cloud the signal after the cloud has been lost. However, a good fitting can be achieved with the part of the signal from above the cloud.

In Fig. 3 the projection in the focal plane has been represented. To obtain the value of the azimutal angle, one needs just to calculate the arctangent of the slope of this projection. As in the previous case, for the optically thin cloud case (intermediate panel), even if some few photons have been scattered, shower track projection is still well defined. On the other hand, in the case of the optically thick cloud (lower panel) the signal after the cloud has been vanished. However, a good fitting can be achieved with projection of the signal from above the cloud.
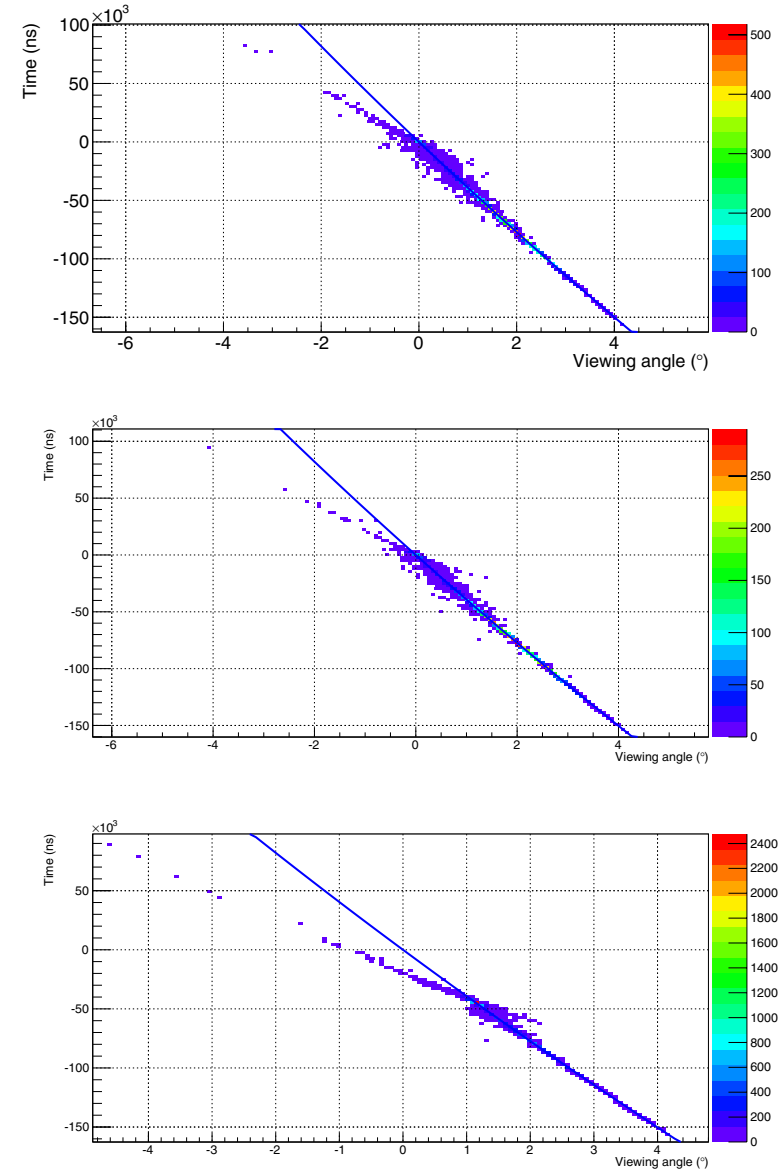

Figure 2. Viewing angle as a function of time for three different cases: in clear atmosphere (upper panel), in presence of an optically thin cloud (intermediate panel), and in presence of an optically thick cloud (lower panel). The slope of the fit is the so called angular velocity. The color palette indicates the number of photons detected by each pixel.

Thus, we can assume that angular reconstruction is feasible for the previously called "reconstructible" cases (i.e., the cloud is optically thin with $\tau<1$, or the altitude of the shower maximum is higher than the cloud top height, $H_{\text {max }}>H_{c}$ ).

Nevertheless, in presence of optically thick clouds the angular resolution is modified. Since the signal after the cloud is totally lost, the EAS shower track will be shortened, and therefore the angular resolution for an EAS in presence of an optically thick cloud will be equivalent to that of an EAS in clear atmosphere with a lower zenith angle. On the other hand, the apparent movement of an EAS in presence of an optically thin cloud will not be significantly modified, since the lenght of the shower track and its timing does not change. Therefore, the angular resolution will be similar to that of the same EAS in presence of clear atmosphere.

\section{Cloud top height retrieval algorithm}

We can parametrize the total extinction coefficient $(\epsilon)$ in terms of the Ice Water Content (IWC) and on the 

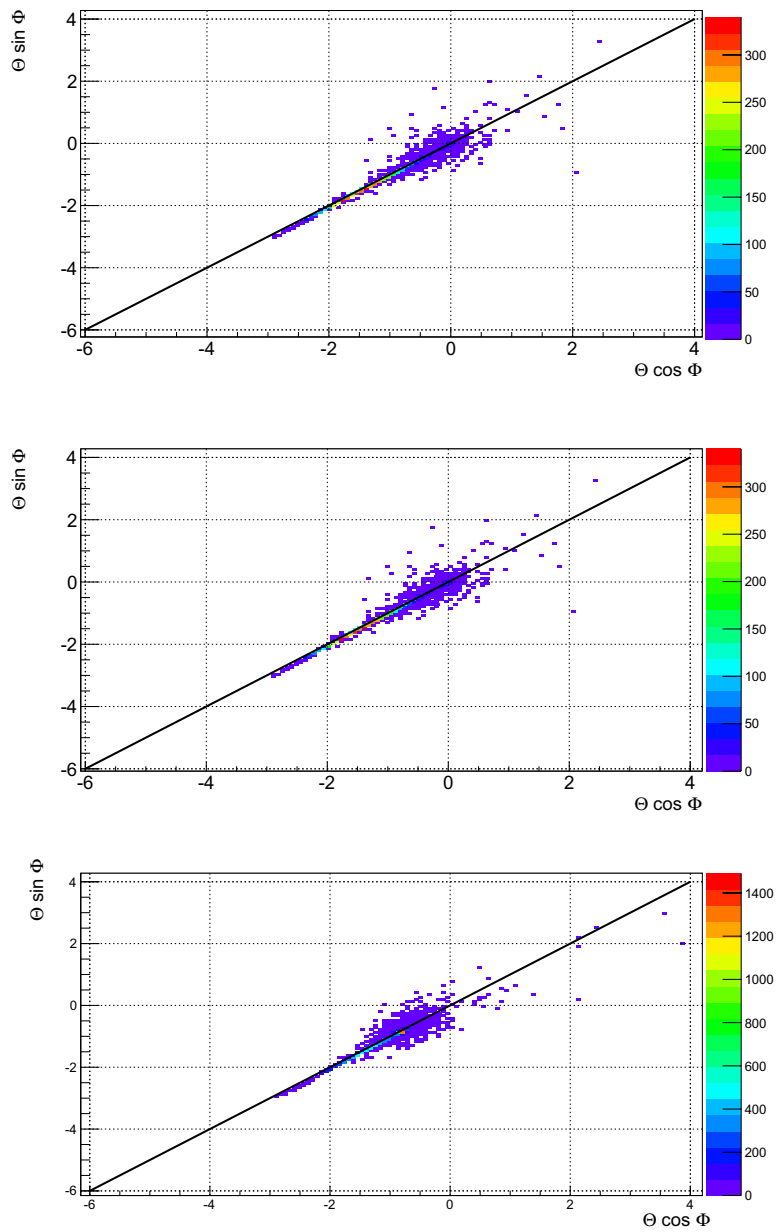

Figure 3. Projection in the focal plane of the three previous cases: in clear atmosphere (upper panel), in presence of an optically thin cloud (intermediate panel), and in presence of an optically thick cloud (lower panel). With the arctangent this projection's slope, the azimutal angle is derived. The color palette indicates the number of photons detected by each pixel.

fixed ice size distribution [8]. Being $\beta_{\text {ground }}$ the irradiance of the ground at a certain temperature, and $\beta_{\text {cloud }}$, the irradiance of the cloud at another temperature (following an atmospheric temperature profile), we can calculate the detected irradiance by the IR-Camera as:

$$
\beta=\beta_{\text {ground }} \cdot(1-\epsilon)+\beta_{\text {cloud }} \cdot \epsilon .
$$

In Fig. 4 it has been plotted, for a cloud of $500 \mathrm{~m}$ thickness at $3 \mathrm{~km}$ altitude, how the integrated extinction coefficient in the near infrared varies with the ice water content of the cloud. Another cloud, has been modeled at $5 \mathrm{~km}$, and the reconstruction performance is calculated for both cases. In this paper, we have used an US-STD atmosphere temperature profile.

\subsection{LIDAR simulation}

For the same ice water content and the same fixed ice size distribution, we have simulated the extinction coefficient for the LIDAR wavelength, and the backscattered signal

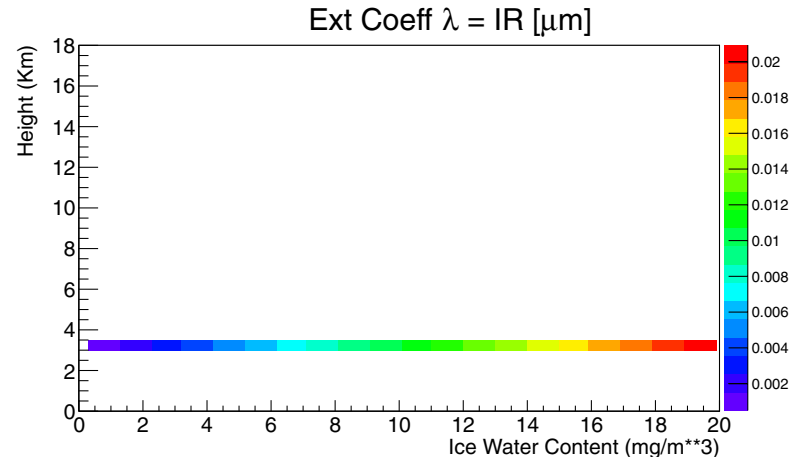

Figure 4. Example of a cloud of $500 \mathrm{~m}$ thickness at $3 \mathrm{~km}$ altitude ( $\mathrm{Y}$ axis). The figure represents the integrated extinction coefficient in the near infrared (color scale) depending on the ice water content ( $\mathrm{X}$ axis).

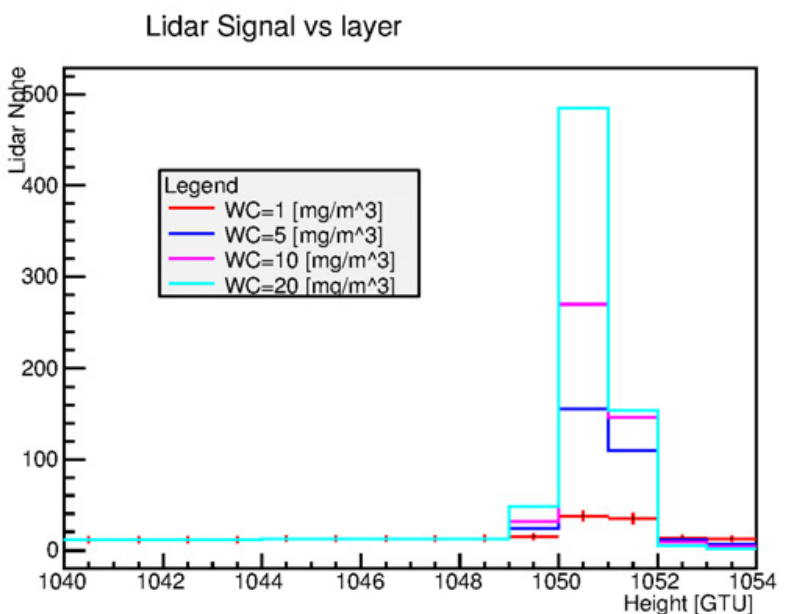

Figure 5. Representation of the simulated backscatter LIDAR signal, at different IWC concentrations. Notice how the signal its spread for several GTU, this leads that at different concentrations, and noise levels, the algorithm to detect the cloud from this signal has a resolution not lower than 2 GTU.

that JEM-EUSO would detect to understand the capability of the LIDAR to measure the cloud optical depth [7]. Figure 5 shows an example of the simulated backscatter LIDAR signal, at different IWC concentrations for a cloud of $3 \mathrm{~km}$ altitude and a physical thickness of $500 \mathrm{~m}$. In our simulation, the LIDAR resolution would be \pm 2 GTUs, which corresponds to an altitude of $1500 \mathrm{~m}$ for a LIDAR signal that has been shot vertically. A more comprenhensive simulation and analysis of both LIDAR, and Infrared Camera is under developement.

To model the atmosphere we have considered an hidrostatic approximation expressed in Eq. (6):

$$
\frac{d \rho}{d z}=-\frac{\rho}{g}
$$

Where $\rho$ is the density, $z$ is the height and $g$ is the gravity constant. If we integrate last equation, and replace $\rho$ with the ideal gas law definition $p=\rho \times R / M \times T$, the pressure can be written as:

$$
p=p_{0} \times e^{\frac{M \cdot g \cdot z}{R \cdot T}}
$$




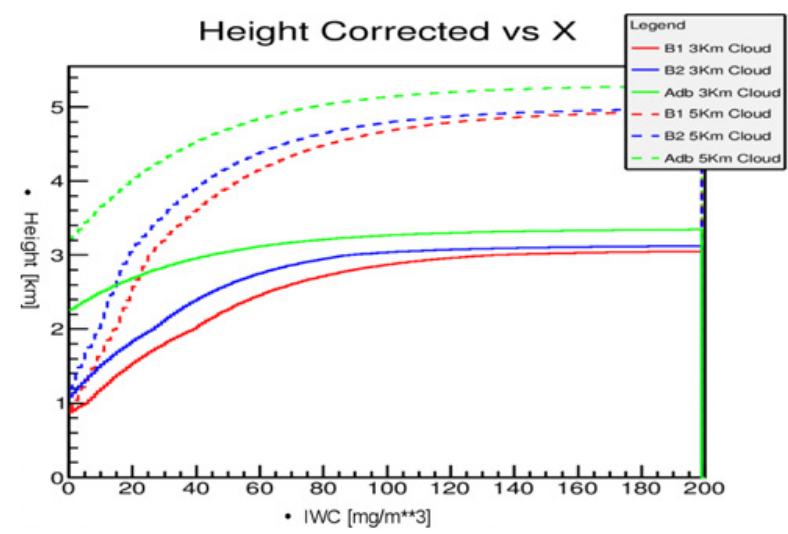

Figure 6. Retrieved height using the proposed abiabatic algorithm of a cloud at $3 \mathrm{Km}$ and $5 \mathrm{Km}$. Comparison with the reconstructed height from the brightness temperature in the $\mathrm{B} 1=$ $10.8 \mu \mathrm{m}$, and $\mathrm{B} 2=11.5$ following the same atmospheric profile used in the simulation is presented as well.

where $p$ is the pressure, $T$ is the temperature of the gas, $g$ is $9.80665 \mathrm{~m} / \mathrm{s}^{2}, M$ the air molar mass is $0.02897 \mathrm{~kg} / \mathrm{mol}$, $R$ the constant gas is $8.3144621 \mathrm{~J} /(\mathrm{mol} \times \mathrm{k})$ and $p_{0}$ is the pressure at sea level that corresponds to $101325 \mathrm{~Pa}$ [8].

Therefore, we define the atmosphere pressure under the adiabatic aproximation as

$$
p \times T^{\frac{(\kappa-1)}{\kappa}}=\text { Constant }
$$

where $\kappa$ is the air heat capacity ratio, fixed as $7 / 5$, considering the atmosphere a diatomic gas of only oxigen, and nitrogen. Combining the pressure equation in a gas enviroment, like the atmosphere, with the last equation of the adiabatic aproximation we obtain a temperture-height relation.

As stated in the previous section, the LIDAR will provide us the altitude of the cloud area seen by a certain pixel, where the LIDAR has been shot. Then we use this information, together with the temperature of the cloud retrieved by the Infrared Camera, to calculate the adiabatic constant that we need in our model. To find out the altitude of the cloud seen by the rest of the IR camera pixels, we apply the adiabatic model with the obtained adiabatic constant and we use the temperature of the different pixels. In the results presented in this paper, we have simulated a LIDAR shoot at an IWC $=100 \mathrm{mg} / \mathrm{m}^{3}$, then calculate the adiabatic constant and reconstruct the rest of the cloud.

\section{Summary and conclusions}

JEM-EUSO is an space based telescope which will use the atmosphere to detect the Extensive Air Showers (EAS) produced by the Ultra High Energy Cosmic Rays (UHECR) when they interact with atmospheric particles. Since UHECR have a very low flux, it is important to increase the exposure of the telescope as much as possible. In this paper we prove how the arrival direction for events in presence of optically thin clouds is similar to that of the same event occurred in a clear atmosphere. Also, it is explained why it is possible to obtain the arrival direction of an EAS in presence of an optically thick cloud, if the shower maximum is visible, with a slightly worse resolution than the one we would get for the same event in a clear atmosphere.

To use events in cloudy conditions for our analysis, it is extremelly important to measure the properties of the clouds in the FoV of JEM-EUSO. Moreover the radiometric retrieval algorithm that fulfills the technical requierements of the IR Camera of JEM-EUSO to obtain the top cloud height from the temperature measured by the IR camera, using a single LIDAR shot for calibration is reported. However, as showed in this analysis, it is clear that the adiabatic method aproximation is far more accurate, than the use of the brightness temperature and the atmosphere profile.

The JEM-EUSO team at the University of Geneva acknowledges support from the Swiss Space Office through a dedicated PRODEX program. This work is supported by the Spanish Government MICINN \& MINECO under projects projects AYA2009-06037-E/AYA, AYA-ESP 2010-19082, AYA-ESP 2011-29489-C03, AYA-ESP 2012-39115-C03, AYA-ESP 201347816-C4, MINECO/FEDER-UNAH13-4E-2741, CSD200900064 (Consolider MULTIDARK) and by Comunidad de Madrid under projects S2009/ESP-1496 \& S2013/ICE-2822. The calculations were performed using the RIKEN Integrated Cluster of Clusters (RICC) facility \& the Space and Astroparticle SPASUAH Cluster. J.A. Morales de los Rios wants to acknowledge the financial support from the UAH-FPI grant and the RIKENIPA program. M. D. Rodriguez Frias acknowledge International Visitor Grant from the Swiss National Science Foundation (SNSF).

\section{References}

[1] Rodríguez Frías, M. D. et al. for the JEM-EUSO Collaboration. The Atmospheric Monitoring System of the JEM-EUSO Space Mission. Proc. International Symposium on Future Directions in UHECR Physics, The European Physical Journal, Vol. 53, 10005pg1-7, http://dx.doi.org/10.1051/epjconf/ 20135310005, (2013)

[2] The JEM-EUSO Collaboration (corresponding authors S. Toscano, J. A. Morales de los Rios, A. Neronov, M. D. Rodríguez Frías \& S. Wada). The Atmospheric Monitoring System of the JEM-EUSO instrument. Experimental Astronomy 37, DOI: 10.1007/s10686014-9378-1 (2014)

[3] Sáez-Cano, G. et al. for the JEM-EUSO Collaboration, "Observation of ultra-high energy cosmic rays in cloudy conditions by the space-based JEM-EUSO Observatory", J.Phys.Conf.Ser., 375, 2012

[4] Sáez-Cano, G., Shinozaki, K., del Peral, L., Bertaina and M. and Rodríguez Frías, M.D. for the JEMEUSO Collaboration, "Observation of extensive air showers in cloudy conditions by the JEM-EUSO Space Mission", Advances in Space Research, 53, 1536-1543 (2014)

[5] Adams Jr., J.H. et al. (The JEM-EUSO Collaboration), An evaluation of the exposure in nadir observation of 
the JEM-EUSO mission. Astroparticle Physics, 44, 76, 90 (2013)

[6] Abu-Zayyad, Tareq and Jui, C.C.H. and Loh, E.C., "The effect of clouds on air showers observation from space", Astropart.Phys., 21, 163-182 (2004)

[7] Rodríguez Frías, M.D. Toscano, S., Bozzo, E., del Peral, L., Neronov, A., and Wada, S. and for the JEM-EUSO Collaboration. The Atmospheric Monitoring System of the JEM-EUSO Space Mission. These Procc

[8] Fu, Q. and Liou, K.N., "Parametrization of the Radiative Properties of Cirrus Clouds", Journal of Atmospheric Sciences, 50, 2008-2025p, 1993 